\title{
Ulcus neuroticum mucosae oris. (Chronische Aphthen.)
}

\author{
Von \\ Dr. J. Löblowitz, \\ Spezialarzt für Hautkrankheiten in Olmütz.
}

In der amerikanischen und englischen Literatur findet sich ein Krankheitsbild, als dessen Charakteristika angegeben werden 1. Geschwürseffloreszenzen auf der Schleimhaut des Mundes, die während mehr minder langer Zeit rezidivieren, 2. mehr minder starke Störungen im Bereiche des zentralen Nervensystems; diese beiden Symptomenkomplexe sollten in einem ursächlichen Zusammenhange zu einander stehen, weshalb diese Geschwüre als "neurotic ulcers", "ulcera neurotica" bezeichnet wurden.

In der deutschen Literatur konnte ich diese Bezeichnung nicht nachweisen; das ihr zugrundeliegende Krankheitsbild ist aber gewiß auch auf dem Kontinente zu finden; da es zuweilen ein sehr schweres Leiden darstellt, so ist des weitern als sicher anzunehmen, daß die Träger desselben ärztliche Hilfe aufsuchen; es kann also den Ärzten nicht unbekannt sein. nur ist es wahrscheinlich, daß diese hiefür eine oder vielleicht auch mehrere anderslautende Bezeichnungen gebrauchen. Da ich Gelegenheit hatte, bei mehreren Mitgliedern einer Familie durch mehr als ein Jahrzehnt ein Krankheitsbild zu beobachten, das mit der von den Amerikanern und Engländern als „neurotic ulcer" benannten Affelstion übereinstimmte, interessierte es mich nachzuforschen, hinter welchem Namen sich diese Krankheit bisher in der deutschen Literatur ver- 
steckte und ob die Bezeichnung derselben als Ulcus neuroticum den tatsächlichen Verhältnissen entspricht.

Bevor ich jedoch in der Besprechung dieser Fragen weiter eingehe, will ich die Krankengeschichte dieser beobachteten Fälle ausführlich mitteilen:

Die Mutter der Familie zeigte die in Rede stehende Affektion niemals und starb an den Folgen einer Otitis media (Sinusthrombose, Diabetes); der Vater hingegen soll seit seiner Kindheit zeitweise an Geschwürsprozessen im Munde gelitten haben, die erst im Alter zwischen $30-40$ vollständig geschwunden seien. Wie ich den Herrn kennen lernte, war er etwa 50 Jahre alt und litt an einem ziemlich starken nervösen Magendarmleiden; dasselbe dauerte schon an die $20 \mathrm{Jahre;} \mathrm{ob} \mathrm{dieses}$ Leiden von Beginn an nervöser Natur war oder sich, was wahrscheinlicher ist, erst im Anschlusse an einen aluten Magenkatarrh angeschlossen hat, ließ sich nach so langer Zeit natürlich nicht mehr sicherstellen; da es sich tatsächlich um ein Leiden auf nervöser Grundlage, wenigstens später, gehandelt hat, beweist die Tatsache, dal er nach jedem Kuraufenthalte in Karlsbad in schlechterem Zustande nach Hause kam, sich dagegen nach Kaltwasserbehandlung in Gräfenberg wobler füblte; er starb im Alter von 56 Jahren an Herzschlag (Arteriosklerose der Koronargefäße).

Von den 7 Kindern (kein Abortus) starb eines im 2. Lebensjahre an Scharlach, das älteste (eine Frau) mit 30 Jahren an den Folgen eines vitium cordis (Gehirnembolie mit Jacksonscher Epilepsie, Gelenkrheumatismus im Alter von 12 Jahren); ein Mädchen zeigt seit seiner Kindheit (13. Lebensjahre) Zeichen einer schweren Neurasthenie, die sich in mehrjährigen Intervallen mit starken, oft monatelang dauernden Depressionszuständen (Melancholie) vergesellschaftet; für gewöhnlich ist die Neurasthenie mehr auf gewisse Organe beschränkt: Magen und Darm (hochgradige Obstipation), ferner Kopf (Kopfdruck, Druck auf die Augen mit einseitigem Tränenträufeln, Druckschmerzhaftigkeit an den Austrittsstellen des $\mathrm{n}$. infra- und supraorbitalis etc.); die übrigen Geschwister zeigen bis auf die Zeichen eines zeitweise leicht erregbaren Temperaments keinerlei hervorstechende nervöse Erscheinungen. Die Frauen der Familie leiden an sehr profusen, 1 Woche und länger dauernden Menorrhagien und bis auf eine auch an starken Dysmennorrhoen, welche sie körperlich oft so herunterbringen, daB sie sich in der Zwischenzeit kaum erholen können.

Was nun die bei den Mitgliedern dieser Familie zu benbachtende Mundaffektion betrifft, so tritt dieselbe bei allen Geschwistern, sowohl männlichen als weiblichen auf, bei letzteren allerdings in weitaus stärkerem Grade; während die Männer nur hie und da diese Geschwüre akquirieren (1-2mal im Jahre), leiden die weiblichen Familienmitglieder, und unter diesen wieder die jüngsten, besonders stark unter dieser Affektion; diese Ietztere belömmt fast das ganze Jahr derartige Mundgeschwüre; diese bäufen sich namentlich zur Zeit der Menstruation in einem solchen 
Maße und sind so groß und schmerzhaft, daß das Kauen und Sprechen auß3erordentlich behindert sind; es leidet die Nahrungsaufnahme und die Patientin zeigt nach einer solchen Attacke Zeichen von großer körperlicher Schwäche, nervöser Reizbarkeit etc.

Die Affektion scheint vornehmlich dem jugendlichen Alter eigen zu sein, um in den dreiBiger Jahren von selbst zu schwinden; Jahreszeit und Witterung haben keinen Einfluß auf die Krankheit.

Unter den Geschwistern des Vaters konnte ich eine Schwester eruieren, die angeblich ebenfalls in ihrer Jugend von zeitweise auftretenden ulzerösen Prozessen auf der Mundschleimbaut heingesucht wurde, bei 2 von ihren Töchtern habe diese selbst gesehen und mit den bei den Mitgliedern der erstgenannten Familie vorkommenden Fälen identifizieren können, doch traten die Anfälle nur selten und in geringem Grade auf, so daß sie sich erst auf Befragen daran erinnerten. Anfall):

Status und Verlauf einer Ulcus aphthosumeffloreszenz (mittelschwerer

„Der Verlauf eines solchen Geschwüres, das ich des ofteren vom Beginne an verfolgen konnte, ist ein typischer, gleichmäßiger, nur in bezug auf die Intensität wechseInder; der Pat. fühlt, sofern die Stelle mit der Zunge erreichbar ist, ein Knötchen auf oder vielmehr unter der Schleimhaut der Wangen, Lippen, das ohne eine ihm bekannte Ursache und schmerzlos auftritt; dasselbe vergrößert sich durch 1-2 Tage und meist unter leichten Fiebererscheinungen - sind gleichgeitig mehrere und größere Effloreszenzen vorhanden, dann steigt das Fieber auf $39^{\circ}$ und mehr - entwickelt sich an der Spitze des halbkonvexen Hügelchens ein sich rasch vergrößerndes Geschwür; ich hatte des öftern Gelegenheit, diesen Übergang aus entzündlicher Infiltration in Ulzeration zu beobachten; letzterer stellt sich dar als ein unregelmäßig, aber scharf begrenzter Substanzverlust von Linsen- bis Bohnengröße, der mit einem grauweißlichen, nicht abwischbaren, speckigen Belage bedeckt ist; es besteht ein ziemlich hoher Infiltrationswall mit mehr minder breiten Entzündungshof. So ist der Befund in einem mittelsehweren Anfalle; bei einer schweren Attacke, besonders wenn die Ulzera an Stellen auftreten, die dem Drucke der Nachbarorgane ausgesetzt sind, z. B. hinten und außen vom letzten Molaris, auf der Zunge etc. verändert sich das Krankheitsbild ganz bedeutend; die Geschwüre werden bis kreuzergroß, vertiefen sich kraterförmig; Infiltrationswall und Entzündungshof erreichen eine ungewöhnliche Größe. Der dicke Belag verwandelt sich durch sekundäre Infektion mit Fäulnisbakterien (Plaut-Vincentsshen Bazillen u. a.) in eine bräunliche, morsche, äußerst stinkende Masse; die Temperatur steigt auf $40^{\circ}$, die entsprechenden Lymphdrüsen sind entzündlich vergrößert und druckschmerzhaft. Es besteht SpeichelfuB, desquamativer Katarrh der Schleimhaut, Ödem. In jedem Falle, ob leicht oder schwer, ist die Schmerzhaftigkeit eine außerordentlich große. Bei schweren Anfällen ist der Zustand der betreffenden Kranken ein geradezu qualvoller: Jede Bewegung beim Sprechen, Essen, Lachen verursacht solche Beschwerden 
daB die Pat. es vorziehen gar nicht zu essen und zu trinken; sehr unangenehm ist auch die dabei eintretende Salivation.

Nach 2-5 Tagen, in schweren Fällen erst nach 1-2 Wochen stößt sich der eitrige, bzw. gangränöse Belag ab und hinterläßt, je -nach der Intensität der vorangegangenen Ulzeration einen oberflächlichen oder tiefen Substanzverlust; der sich bald mit gesunden Granulationen ausfüllt; die Narben sind meist nur kurze Zeit sichtbar. Der ganze Prozeß dauert also in toto $1-3$ Wochen. - Was di e $\mathrm{Z}$ ah $\mathrm{l}$ der Geschwürseffloreszenzen betrifft, so beobachtete ich meist nur ein Ulkus, selten 2 oder mehrere (bis zu 6 gleichzeitig), was immer Zeichen eines schweren Anfalles und mit großen subjektiven Beschwerden verbunden ist.

In bezug auf den Sitz der Affektion läßt sich sagen, daß die Ulzera an allen Stellen der Mundschleimhaut auftreten können, zumeist sah ich sie aber auf den Lippen, Wangen und Zunge; ob die auf den Tonsillen zuweilen beobachteten Geschwüre auch in dieselbe Kategorie zu rechnen sind, wie die eben beschriebenen, Jäßt sich in den Fällen, wo sie gleichzeitig mit letzteren vorhanden waren, als wahrscheinlich annehmen; bei alleinigem Sitz auf den Tonsillen ist dagegen die Diagnose schwieriger, denn bier läßt sich der typische Verlauf wie bei Sitz auf der übrigen Schleimbaut, nicht nachweisen; sie ließen sich gegen die Angina necrotica anderer Herkunft, gegen die Angina Vincent nicht oder wenigstens nicht mit Sicherheit abgrenzen; der Sitz der Geschwüre ist für den Verlauf derselben nicht ohne Bedeutung; denn die bösartigsten und schmerzhaftesten fanden sich an Stellen, wo die entzündete Schleimhaut dem Drucke der Nachbarorgane ausgesetzt sind, z. B. an Kjeferwinkel; hinten und außen vom letzten Molaris, an den Zungenrändern; besonders im hintern Anteil usw:

Die mikroskopische Untersuchnng des Geschwürsbeleges in beżng auf die Bakterienflora ergab keinen Anbaltspunkt für einen etwaigen spezifischen Erreger; es fanden sich "nur die gewöhnlichen Eitererreger; Staphylo- und Streptokokken, kurze, dicke Stäbchen, selten längere, etwas gebogene Stäbchen und vereinzelte große Spirochaeten mit wenigen und unregelmäBigen Windungen. In den Fällen, wo der Belag morsch und foetide riechend befunden wurde, lieben sie die von $\mathrm{Pla}$ l und später von $\mathrm{V}$ in cent genauer beschriebenen Bazillen und Spirillen fast in Reinkultur nachweisen. Es machte nicht den Eindruck, als ob diese genannten Bakterienbefunde irgend eine ätiologische Bedeutung für die Entstehung dieser Vlzerationen haben würden, vielmehr scheinen alle dieśe Mikroben erst sekundär eingewandert zu sein; leider war es mir nicht gestattet, die Exzision einer Geschwürseffloreszenz vornehmen zu dürfen; so muBte die histologische Untersuchung nnterbleiben; dieselbe wäre namentlich im Entwicklungsstadium noch vor der Exulzerierung von Interesse und vielleicht anch von Bedeutung gewesen.

Fassen wir den Inhalt dieser etwas ausführich wiedergegebenen Krankengeschichte kurz zusammen, so handelt es sich um eine chronische ulzerative Affektion der Mundschleimbaut, die 
entschieden hereditär und familiär auftritt und sich charakterisiert durch das mehr minder häufige Auftreten von umschriebenen Geschwürseffloreszenzen von bestimmtem klinischen Charakter; die Affektion ist eine Krankheit des jugendlichen Alters und nimmt im mittleren Lebensalter der betreffenden Individuen allmählich an Intensität $a b$; sie scheint eine Folge sehr verschiedener Schädlichkeiten zu sein, deren Gemeinsames darin besteht, daß sie den Organismus weniger widerstandsfähig machen g e gen über den Angriffen sonst nicht pathogener Keime z. B. profuse, stark schwächende Menstruationsblutungen, Schwangerschaften, die Zeit der Pubertät etc. Jedenfalls ist das N ervensystem in hohem Grade mitbeteiligt und es wird Sache späterer Auseinandersetzungen sein festzustellen, ob diese Beteiligung primärer oder nur sekundärer Natur ist.

D ifferentialdiagnostisch kommen alle jene Affektionen in Betracht, bei denen es zur Bildung von rezidivierenden Geschwürseffloreszenzen auf der Schleimhaut der Mundhöhle kommt; da wäre vor allem das sog. Ulcus folliculare mucosae oris, auch dyspeptisches oder katarrhaliches Mundgeschwür genannt, zu erwähnen; doch sind die klinischen Unterschiede beider Affektionen so bedeutend - das Ulc. follic. ist ein linsengroßes, ganz oberflächliches Errosivgeschwürchen, charakterisiert durch den dünnen, weiblichen Belag inmitten eines ungemein breiten Entzündungshofes ohne Infiltrationswall - ephemeren Charakter etc.

Viel schwieriger ist die Differentialdiagnose gegenüber den u lzerö sen Syphiliden der Mundschleimhaut, die ebenfalls zeitweise auftretende Ulzerationsprozesse auf der Mundschleimhaut veranlassen; in der Tat haben die Aphtheneffloreszenzen grobe Ähnlichkeit mit gewissen Formen der Lues und bei einer nur einmaligen oder ungenügenden Untersuchung würde wohl jeder die Diagnose auf Syphilis stellen, besonders wenn die Anamnese nicht als einwandfrei sich herausstellen sollte. Gegen diese Diagnose in meinen Fällen spricht außer der äußerlichen Ähnlichkeit alles. Weder läßt sich in der Anamnese irgendein Anhaltspunkt für Lues eruieren, noch zeigen oder zeigten die betreffenden Personen sonstige luetische oder hereditärluetische Symptome an anderen Organen; die Frauen dieser Familie, auch 
ein Bruder derselben sind alle schon lange Zeit verheiratet, niemals ein Abortus zu konstatieren, auch an deren Kindern war von Lues nichts zu konstatieren. - Auch die Geschwüre selbst unterscheiden sich bei väherem Zusehen von den syphilitischen durch den typischen Beginn als rotes Hügelcher, das mit einer grauen Plaque muqueuse keine Ähnlichkeit zeigt, durch ihren stark ausgeprägten breiten Infiltrationsring gegenüber dem ganz schmalen, dunkelroten charakteristischen Wall beim ulcus syphiliticum, durch seine überaus große Schmerzhaftigkeit, während die luetischen Effloreszenzen im allgemeinen nicht sehr schmerzen oder doch nur ausnahmsweise sehr große Schmerzhaftigkeit erregen.

Allerdings die grauen diphtheroiden Beläge der Frühperiode (errosives Stadium) und der schmierig-speckigen, leicht abwischbaren Beläge der späteren Perioden, wo auch die Frruptionen isoliert stehen und stärkere Tiefeninfiltration aufweisen, unterscheiden sich sehr wenig von den Belägen des Ulcus aphthosum, so daß eine Verwechslung sehr leicht möglich ist, wenn man nicht die Krankheitssymptome anderer Organe in Erwägung zieht. Differential-diagnostisch wäre noch zu erwähnen, daß das Ulcus aplithosum gegenüber den therapeutischen Maßnahmen: Lapis, Chromsäurepinselungen, die auf luetische Geschwüre sehr günstig einwirken, gar nicht reagieren und wegen der allzu großen Schmerzen, die sie verursachen, sehr bald energisch abgelehnt werden. Ferner ist auf die Tatsache binzuweisen, die man allerdings erst nach längerer Beobachtungszeit feststellen kann, daß es nach allgemeiner Erfabrung kaum Fälle von Lues gibt, wo sich die Effloreszenzen des II. Stadiums durch mehr als ein Menschenalter ungeschwächt wiederholen würden.

Sehr große Ähnlichkeit, nicht sowohl in bezug auf die Effloreszenzen als bezüglich des Verlaufes, Dauer des Leidens, event. auch die Ätiologie der ganzen Krankheit-besteht zwischen dem Ulcus aphthosum und dem $\mathrm{Herpes}$ buccalis, besonders jener Form, die als Herpes recidivans Fournier bezeichnet wird; auch bei dieser Krankheit findet sich jahrzehntelange Dauer der Affektion, sehr häufige Rezidiven (alle 2-8 Wochen), auch hier bei den Kranken Anzeichen mehr minder starker Störungen des Nervensystems. Der Unterschied zwischen beiden 
Affektionen zeigt sich aber bei den Einzeleffloreszenzen, namentlich in den ersten Stadien. Das Ulcus neuroticum beginnt als umschriebenes, ziemlich derbes Hügelchen, das an der Kuppe zu einem einzigen Geschwürchen zerfällt, das von einem hohen Infiltrationsringe eingeschlossen ist; der Herpes beginnt auf der Schleimbaut auch mit einer mehr oder minder hohen Schwellung; dieselbe ist aber weich, ödematös und darauf entwickelte sich eine Gruppe von Vesikeln; wenn diese geplatzt sind, was sehr bald eintritt, dann sieht man die polyzyklisch begrenzten pseudomembranösen Beläge (Blasendecke) oder deren Reste (Kollerrette) und nach Abfall derselben die bloBliegende Erosion; wenn sich diese durch sekundäre Infektion in Geschwüre verwandeln, die vielleicht an günstiger Stelle sich auch kraterförmig tief in die Schleimbaut erstrecken, ist allerdings die Ähnlichkeit eine sehr große; in einem solchen Falle dürfte aus der Effloreszenz allein die Diagnose nicht zu stellen sein.

Sonstige ulzeröse Prozesse der Mundschleimhaut, so die tuberkulösen u. a. kommen differential-diagnostisch nicht ernstlich in Betracht.

Jie Affektion, um die es sich bei meinen Fällen handelt, stellt ein so auffallendes, in die Augen fallendes Krankheitsbild dar, daß es unwahrscheinlich erscheint, daß es bisher in deutschen Landen nicht beobachtet und beschrieben sein sollte; mir ist es allerdings nicht gelungen, in der deutschen Literatur eine ausführlichere gleichartige Krankengeschichte nachzuweisen, wobl aber in der amerikanischen und englischen, wo die Affektion als Ulcus neuroticum beschrieben wird, die ganz genau mit der von mir beobachteten übereinstimmt, wie wir später noch sehen werden; ich glaube daher vielen Grund zu der Annahme zu haben, daß dieser ulzeröse Prozeß bei uns bisher jenem Krankheitsbegriffe subsumiert wurde, das gemeinhin als chronische Aphthen der Mundschleimhaut bezeichnet wird; es ist wohl möglich, daß dies der Fall ist - wenigstens haben die Kollegen, welche die Affeltion zu sehen Gelegenheit hatten, wie z. B. Prof. Spiegler, sie als solche aufgefaßt - aber gewiß ist es auch, daß dieser Name: Ulcus aphtbosum chronicum bisher kein einheitliches Krankheitsbild darstellte; vielmehr wurde und wird er noch heute von den verschie- 
denen Autoren zur Bezeichnung sehr verschiedener ulzeröser Schleimhautaffektionen der Mundhöhle in Anspruch genommen, die gar nichts miteinander gemein haben; er ist zu einem Sammelnamen geworden, der Ulzerationen sehr verschiedener Provenienz in sich schließt; besonders ist dies in der älteren Literatur der Fall, wo auch nicht geschwürige Prozesse darunter verstanden wurden, z. B. Soor etc.; erst nach und nach schälten sich mit zunehmender Erkenntnis einige Krankheitsbilder als selbständige Affektionen aus und zwar:

1. Die Stomatitis aphthosa epidemica s. str., die echten Aphthen, eine hauptsächlich bei Kindern, selten bei Erwachsenen an der Mundschleimhaut, zuweilen auch an anderen Schleimhäuten (ad genitale) zu findender Entzündungsprozeß, dessen Einzeleffloreszenzen durch ein Exsudat zwischen Epithel und Mukosa gebildet werden, also ein pseudomembranöser Prozeß, nicht eine Ulzeration, die aber in eine solche durch sekundäre Infektion und Eitererregern übergehen kann.

2. Die B edn ař schen A ph the n, symmetrische Dekubitalgeschwüre am harten Gaumen, die bei schon katarrhalisch veränderter Mundschleimhaut kleiner Kinder, wahrscheinlich durch die mechanische Einwirkung des Saugaktes auftreten.

3. Die Stomatitis aphthosa epizootica, Aphthenseuche, auch Maul- und Klauenseuche genannt, eine akute Infektionskrankheit, die vom Tier auf Menschen übergehen kann. Aber auch nach Ausscheidung dieser ulzerösen Affektionen ist der Krankbeitsbegriff: "Chronische Aphthen" noch immer nicht einheitlich bzw. eindeutig; so werden namentlich verschiedene, auf traumatischer Grundlage entstandenen Geschwüre der Mundschleimhaut des öfteren als Aphthen angesprochen (Bresge n), am häufigsten wohl die kleinen, flachen, weißlichen Schleimhautulzerationen mit sehr breitem Entzündungshofe, die ich mit Lesser u. a. als Follikulargeschwüre der Mundschleimbaut bezeichne; diese beiden Prozesse sind ganz verschieden voneinander und haben miteinander nichts zu tun.

Sehen wir von genannten und selbstrerständlich von den auf Tbc. und Lues beruhenden Ulzerationsprozessen der Mundschleimhaut $a b$ und dem daselbst äußerst seltenen Ulcus 
molle, ferner der großen Gruppe der sogenannten „sekundären Ulzerationen", die durch sekundäre Infektion andersartiger Proruptionen entstanden sind (Vesikel etc.), so verbleibt eine Gruppe von Mundgeschwüren, die schon mehr einen einheitlichen Charakter tragen, die man alter Gepflogenheit folgend, wenn auch nicht sehr bezeichnend, unter der Bezeichnung: Ulcus aphthosum chronicum, chronische Aphthen zusammenfassen könnte; zwischen den einzelnen Formen dieser Gruppe bestehen weniger qualitative als quantitative Unterschiede, d. h. sie unterscheiden sich nur durch die Zahl der Rezidiven, Schwere der Affektion etc.

Das Gemeinsame der in dieser Gruppe vereinigten ulzeröser Prozesse ist, daß sie als solide submuköse entzündliche Infiltrate in Erscheinung treten - also wahrscheinlich nicht von einer von außen einwirkenden Noxe berrühren - welche Infiltrate erst im weiteren Verlaufe oberflächlich exulzerieren und ein mehr minder großes und tiefes Ulkus mit scharfgezackten Rändern and hohem Infiltrationswalle darstellen, das mit einem speckigen, festhaftenden, gewöhnlich nicht foetide riechenden Belage bedeckt ist; löst sich dann der letztere, so resultiert ein napfförmiger Substanzverlust, der natürlich nur mit Narbenbildung ausheilen kann; die Narbe ist allerdings auf der Schleimhaut recht bald nicht mehr zu erkennen.

Einzelne solche Geschwüre sind wohl nicht allzu selten; diese leichten Formen, wo nur in großen Zwischenräumen eine oder die andere Effloreszenz auftritt und ohne besondere Beschwerden in einigen Tagen abläuft, werden de norma - außer von Luetikern und Syphilophoben - gar nicht beachtet und können nur durch darauf gerichtete Aufmerksamkeit ron beite des Untersuchers des öftern konstatiert werden. - Anders bei den stark oder stärkstentwickelten Formen; da folgt eine Rezidive der andern auf dem Fuße und eine ist stärker und unangenehmer als die andere, so daß es schwer wird, glaubhaft zu machen, daß diese beiden Formen ein und demselben Krankheitsbilde zugehörig sind; es bestehen also ganz bedeutende In- und Extensitätsunterschiede.

In der eingangs dieser Abhardlung geschilderten Krankengeschichte der Mitglieder einer ganzen Familie liegen nach 
meiner Ansicht leichte und schwere Formen der hier vorläufig als "chronische Aphten" bezeichneten Affektion vor; die schweren Grade darunter zeigen eine bis ins Detail gehende Übereinstimmung mit einem Krankheitsbilde, das von amerikanischen und englischen Autoren als "neurotic ulcer", ulcus neuroticum, bezeichnet wird, so daB ich nicht anstehe, diese beiden Krankheitsbilder miteinander zu identifizieren.

Die Bezeichnung dieser Affektion als ulcus neuroticum stammt von Jacobi (Transactions of the Assoc. of Americ. Physic. vol. IX. 1894), der ausführlich einige Fälle von chronischen Mundgeschwüren (darunter 2 Männer betreffend) beschrieb; die Träger derselben waren schwer nervöse, zum Teil sogar direkt geisteskranke (insaue type) Menschen, weshalb er diese ulzerösen Mundschleimhautaffektionen als auf trophoneurotischer Basis berwhend "ulcera neurotica" benannte. Er führt aus der Literatur, auch der deutschen, alle Fälle an, die seiner Meinung als ulcera neurotica anzusprechen sind, wenn selbe auch von den betreffenden Verfassern unter andern Namen geführt wurden; ich habe dieselben soweit sie mir zugänglich waren, mit meinen Fällen verglichen; Flata u (D. med. Wochenschr, 1891) erwähnt diese Affektion bei Gelegenheit der Differentialdiagnose gegenüber dem Herpes chronic. recid. als charakterisiert durch das Auftreten von soliden Knötchen, die oberfiächlich ulzerös zerfallen und deren speckige Beläge über die Umgebung hervorragen; Mandelstamm (B. Klin. W. 1891: Pemphigus der Mundschleimhaut), Landgraf (ebendasellost); ich mul aber gestehen, daß die von letzteren Autoren heschriebenen Fälle mit den meinigen keine Ähnlichkeit habes, sondern vollkommen der von den Verfassern gestellten Diagnose entsprechen.

Dagegen beschreibt Sibley (Brit. med. Journ. 15. April 1899) 3 Fälle, schwer hysterische, nervöse, aber nicht direkt geisteskranke Frauen betreffend, deren Krankheitsbild mit dem meiner Fälle in den Hauptzügen ubbereinstimmt; er grenat die Affektion ausdrücklich gegen die sog. dyspeptischen und katarrhalischen Geschwüre (ulcus folliculare muc. oris), gegen die Herpes- und Pemphigusprozesse ab, mit denen sie so oft verwechselt wird und faßt sie als direkten Ausdruck einer Trophonkeurose auf.

Im ersten Falle traten die Ulzerationen im 57. Lebensjahre, angeblich als Folge häuslicher Wirrnisse (trouble) auf und dauerten mit kleinern und größern Intervallen, trotz Änderung des Domizils, der Lebensweise und trotz jeglicher Behandlung durch viele Jahre, das Leben der Pat. durch die zahlreichen, überaus schmerzhaften Attacken zu einem qualvollen gestaltend.

Der 2. Fall betrifft eine Frau von 50 Jahren, die von Jugend auf an Mundgeschwüren litt, deren Auftreten mit gewissen häuslichen Zerwürfnissen zu koinzidieren pflegte; sie besaß seit Geburt eine zarte Konstitution und zeigte seit jeher Zeichen ron Neurasthenie, die sich im Klimakterium zu einer Hysteromanie ausbildeten. 
Im 3. Falle handelt es sich um eine 64jährige Witwe, Mutter von 14 Kindern, die ihr viel Arbeit und Sorgen machten; Katamenien im ö3. Jahre. Die Ulzerationen begnanen erst im 61. Jahre mit einem kleinen Knötchen an der Zungenspitze, aus dem sich ein Geschwür entwickelte. Seither bekam sie zeitweise gleiche Effloreszenzen auf dem Gaumen, Zahnfeisch, Seitenränder der Zunge; die Affektion danerte zur Zeit der Niederscbrift dieser Beobachtung noch an und machte ihr zuweilen große Beschwerden.

Kirk (Brit. medic. Journ. 30. Mai 1899) hat ähnliche Fälle beobachtet wie Sibley, doch hält er dieselben nicht für Trophoneurosen sondern bringt sie in Zusammenhang mit Myxödem; die ulzerösen Mundprozesse traten bei seinen Kranken meist schon viel früher auf als die Veränderungen der Thyreoidea; so weist er insbesondere auf einen Fall hin, eine Fran von 40 Jahren betreffend, mit sehr starken Menstruationgblutungen, die während dieser Zeiten zahlreiche Geschwüre auf der Zunge und der übrigen Mundschleimbaut hatte, außerdem aber Schwellungen auf Augenlider und Lippen, weIche das Sprechen sehr erschwerten; Kirk hält diese Symptome für Zeichen von Thyreoideaerkrankung und meint, daß auch 2 von den Sibleyschen Fällen und 1 Fall von Jakobi (l. c.), einen in Wachstum zurückgebliebenen Mann betreffend, der als Kretin aufzufassen ist (er hatte keinen Bart, keine Haare ad pubes und ad axillam; Sexualorgane wie ein Kind etc.), auf Veränderungen der Schilddrüse zurückzuführen sind nnd schlägt daher als rationellstes Mittel die Behandlung mit Schilddrüsepräparaten vor.

Arthur Court (Brit. med. Journ. 30. Mai 1899) veröffentlicht, angeregt durch die Mitteilungen von Sibley und Kirk die Krankengeschichte einer 36jäbrigen Frau, Mutter von 5 Kindern, die früher stets gesund war, weder syphilitische noch tuberkulöse Symptome aufwies; sie litt seit 10 Jahren an chronischen Ulzerationen im Munde, die abheilten und nach einigen Wochen wiederkehrten; die Rezidiven koinzidierten anf Rezidive ibres nervösen Leidens, das sich angeblich als Folge von anhaltenden Sorgen und Angst eingestellt hatte. Die Geschwüre saßen am Gaumen, an der Innenfläche der Lippen, Zunge, ja sogar im Rachen.

Sonst fand ich in der mir zugänglichen Literatur nur noch sehr wenig über diese als Ulcus aphthosum chronicum bezeichnete Affektion; so stellte Freund (ref. im Arch. f. D. u. S. Bd. LIII. p. 164) anläßlich einer Debatte über Ulzerationsprozesse im Munde eine 42jährige Fran vor, die im Frühling und Herbst gleichzeitig mit Menstruationsstörungen Geschwüre auf der Mundschleimhant bekam; diesen pflegten gelbe Bläschen (?) von ErbsengröBe voranzugehen; interessant dabei ist, daß die 13jähr. Tochter der Patientin an einer ähnlichen Affektion litt; es zeigt sich also ein familiärer und hereditärer Typus wie in meinen Fällen; doch bin ich in den Freundschen Fällen nicht sicher, ob es sich mit Rücksicht auf den den Geschwüren vorangehenden Blaseneffloreszenzen nicht um Herpes recidivans Fournier handelt, der in gleicher Weise ver. laufen kann. 
Inwieweit die von Trautmann (Dermatosen und Lues p. 145) erwähnte Stomatitis materna der Alten, die bei Frauen während der Laktation, Gravidität Menses und verschiedenen pathologischen Veränderungen des Uterus auftreten und die Lang unter der Bezeichnung "Stomatitis uterina" zusammenfaßt, mit dem Ulcus aphthos. chron. im Zusammenhange stehen, kann ich leider nicht beurteilen, da ich die mit obigen Bezeichnungen versehener Krankheitsbilder in dem mir vorliegenden $\mathrm{L}$ a $\mathrm{n}$ g schen Lehrb. d. Syphilis 1904 nicht vorfinden kann; ich kann nur sagen, daß diesen Bezeichnungen eine gewisse Berechtigung nicht abzusprechen ist und daB es mir sehr wahrsoheinlich erscheint, daß darunter eine Affektion za verstehen ist, die mit dem Uleus aphthosum chron. identisch ist; denn auch bei meinen Fällen weiblichen Geschlechtes konnte ich konstatieren, daß sie alle insgesamt an profußen Mennorrhagien und Dysmennorrhoen litten, die auch nach meiner Meinung in einem ursächlichen Konnexe mit den Ulzerationsprozessen auf der Mundschleimhaut zu bringen sind; auf diese Frage werden wir übrigens noch ausfübrlich zurückkommen.

Wenn wir das Krankheitsbild des Uleus aphthosum chronicum so selten in der Literatur, namentlich der neueren, erwähnt finden, so hat das seinen Grund darin, daß es sich in den meisten Fällen nur um leichte, den Pat. nur wenig belästigende Anfälle handelt, mit denen derselbe nur ausnahmsweise ärztliche Hilfe in Anspruch nimmt; so schwere Formen, wie ich sie hier beschrieben und in der englischen Literatur als ulcus neuroticum sich finden, sind, wie alle Beobachter übereinstimmend angeben, äußerst selten. Der weitere Grund ist der, daß dieses Krankheitsbild in den Lehrbüchern auch den Spezialwerken, gar nicht oder nur sebr kursorisch erwähnt wird und dann noch zuweilen in einer Art und Weise, die deutlich zeigt, daß der Verfasser in dieser Sache selbst nicht im Klaren ist, so in dem sonst so brauchbaren Buche von Trautmann (1. c) unter Stomatitis aphthosa, wo die chronischen Aphthen in einem Atem mit der akuten infektiösen Stomatitis aphthosa genannt werden, zwei Affektionen, die himmelweit von einander verschieden sind.

DieseVerwechslung, die häuptsächlich durch dieBezeichnung beider Affektionen als Aphthen hervorgerufen wird - denn die Hinzufügung der Epitheta chronicun und acutum reicht nicht aus, da noch immer der Verdacht bestehen kann, als ob die chronischen Aphthen aus den akuten hervorgehen können ließen es angezeigt erscheinen; für das Krankheitsbild der chronischen Aphthen eine andere entsprechendere Bezeichnung zu wählen, etwa wie die Amerikaner und Engländer den Namen: Ulcus neuroticum; diese Bezeichnung hat vieles für sich, wie wir bei der Besprechung der Ätiologie dieses Ulzerationsprozesses noch ersehen werden; es wäre gegen dieselbe nichts einzuwenden, wenn sie vicht nur zur Bezeichnung der schweren, 
sondern auch der leichteren, nicht so sehr in die Augen fallenden Formen derjenigen Geschwürseffloreszenzen der Mundschleimhaut benützt wird, die ich zur Gruppe des Ulcus aphthosum chronicum zusammengefabt und definiert habe.

Bezüglich der Ätiologie des hier in Frage stehenden Geschwürsprozesses des Ulcus neuroticum (chronische Aphthen) finden sich bei den verschiedenen Autoren sehr bedeutend differierende Ansichten; die einen sehen in dieser Affektion nur eine Folge lokaler Reize auf traumatischer Grundlage (mechanischer, chemischer oder thermischer Natur, z. B.Bre s g en: Krankh. u. Behdlg. der Nase-, Mund- und Rachenkrankh., 1896, p. 306 ; da dürfte wohl eine Verwechslung zwischen ätiologischen und auslösenden Momenten statthaben oder dem Autor das Krankheitsbild einer andern ulzerösen Affektion, das der katarrh. oder dyspeptischen Geschwüre - von mir als ulcus folliculare bezeichnet - vor Augen schweben, bei denen traumatische Momente ätiologisch in Frage kommen, wie wir bei der Besprechung dieser Geschwürsform noch ausführlich hören werden; übrigens scheint diese Erklärung selbst dem Autor nicht für alle Fälle auszureichen, denn schon auf p. 310 des obgenannten Buches meint er, daß chronische Aphthen auch infolge Schwangerschafts-, Menstruations- und Verdauungsstörungen auftreten können.

Kirk (l. c.) sieht das ulcus neuroticum in seinen Fällen an als Folge von verschiedenen Schilddrüsenveränderungen, die er bei seinen Kranken fand und meint, daß die Schilddrüse wahrscheinlich in den meisten Fällen die Ursache der Ulzerationsprozesse sei, nur könnten die Veränderungen dieses Organs mit unsern Mitteln nicht immer nachgewiesen werden. In den von mir beobachteten Fällen deutete nichts auf eine krankhafte Veränderung der Schilddrüse, ich habe daher auch keine Veranlassung, an diese Ätiologie zu denken.

Dagegen sprechen sehr viele Momente dafür, daß diese Geschwürsprozesse durch irgendwelche Einwirkung des Nervensystems zustandekommen, die Schwierigkeit besteht nur darin, diesen Konnex herauszufinden. Ja c o bi (l. c.), von dem die Bezeichnung dieser Affektion als Ulcus neuroticum stammt, ebenso Sibley (l. c.) sprechen von einer Trophoneurose ohne nähere 
Angabe; ist darunter die Störung von Nerven zu verstehen, die auf die Ernährung der Gewebe direkt Einfluß nehmen (trophische Nerven s. str.), so muß man von dieser Erklärung insolange absehen, als solche Nerven nicht einwandfrei nachgewiesen sind, was bisher nicht der Fall ist; versteht man unter Trophoneurosen dagegen solche Gewebsstörungen, bei denen überhaupt Nerveneinfluß, insondern natürlich der Vasamotoren, in Frage lommt, dann kann man dieser Bezeichnung zustimmen; in diesem Sinne ist das ulcus neuroticum eine Trophoneurose und zwar sind es die Gefäßnerven, die hier hauptsächlich in Frage kommen; ich rerweise diesbezïglich auf die Arbeiten von $\mathrm{Kreibich}$ und seiner Schule, der in zahlreichen Abhandlungen (Die angioneurotische Entzündung, 1905, und vielen andern) die wohlfundierte Hypothese von den Angioneurosen aufstellt; darnach sollen die angioneurotischen Hautaffektionen (Herpes febrilis, Herpes zoster, neurotische Hautgangrän etc.) "sympathische Reflexneurosen sein bedingt durch eine gesteigerte Erregbarkeit des dominierenden Vasodilatatorenzentrums"; ausgelöst sollten diese Reflexe werden können sowohl durch funktionelle und organische Vorgänge im Gehirn (psychogene Reize), als auch durch von der Haut oder von andern Körperstellen herrührende afferente Reize, die mit den psychogenen Reizen auf gleicher Stufe ständen. Man mag über die theoretische Begründung dieser Hypothese, welcher Meinung immer sein, Tatsache ist, daß sie sehr vieles bei diesen genannten Affektionen unserem Verständnisse nahe bringt, was nach den bisherigen Lehren unerklärlich blieb. Was dieser Autor in bezug auf Ätiologie des Herpes febrilis und Herpes zoster cutis sagt, das kann ohne weiteres auch zur Erklärung des Herpes mucosae oris (Herpes chron. rezidivans Fournier) herangezogen werden. Das Ulcus neuroticum (chron. Aphthen) wäre dann in Analogie zu bringen zur neurotischen Hautgangrän. Erinnern wir uns des eingangs geschilderten Verlaufes einer Effloreszenz von Ulcus neuroticum, so finden wir klinisch die Phasen einer echten neurotischen Gangrän, wie sie Kreibich an der Haut schildert, wieder: das Stadium der dilatatorischen Hyperämie bekommt man allerdings niemals zu Gesichte, sondern erst das Stadium der submukösen Knötchenbildung, das sich per analogiam durch die 
Gewebsnekrose mit Exsudatdurchtränkung erklären läßt; die weitere Entwicklung des Knötchens zum Ulkus ist dann leicht verständlich; die nekrotische Stelle bildet einen locus minoris resistentiae, auf dem sich dic banalen Eitererreger der Mundhöhle ansiedeln. Auch die Tatsache, daß diese Geschwüre an solchen Stellen, die einem Drucke ausgesetzt sind, z. B. von seiten der Zähne, besonders tiefgreifend sind und ein kraterförmiges Aussehen gewinnen, läBt sich nach $\mathrm{Kreibich}$ dadurch erkiären, daß die pathologische Innervation auf eine bereits irritierte (durch Druck) Gefäßwand stattfindet und daher viel intensivere Wirkung erzielt.

Kreibich nimmt als Grundlage seiner Theorie von den Angioneurosen, wozu ich also auch das Ulcus neuroticum zäblen möchte, an, daß das Vasadilatatorenzentrum durch funktionelle (Hysterie) oder organische Erkrankungen des zentralen Nervensystems (Gliosis spinalis, Tabes etc.) oder event. auch Autointoxikation (Ovarium) in seiner Labilität gestört, gesteigert erregbar sei und daher auf die verschiedenen peripheren sensiblen Reize oder durch zerebrale Vorgänge in ganz abnorm heftiger Weise reagiert; durch diese heftigen, verhältnismäßig rasch sich abspielenden Reaktionen erklären sich nach $\mathrm{Kreibich}$ auch die Schwere der angioneurotischen Hautsymptome: der plötzlichen Hyperämie mit rasch einsetzender Transsudation, die ihrerseits durch Druck auf die Gefäße Anämie mit Nekrose und Exsudatdurchtränkung zur Folge haben.

Ganz in derselben Weise läßt sich die Entstehung des Ulcus neuroticum der Mundschleimhaut erklären, indem sich die eben geschilderten Vorgänge statt auf der Haut auf der Schleimhaut abspielen.

Wir müßten ferner annehmen, daß die Labilität des Vasamotorenzentrums bei den Mitgliedern der von mir geschilderten Familie auf einen familiären und hereditären Zustand hinweist, der sich hauptsächlich in der ersten Hälfte des Lebens deutlicher zeigt, um später allmählich an Intensität abzunehmen; bei einer solchen angeborenen stärkeren Erregbarkeit des genannten Zentrums würden dann die verschiedensten Reize von der Peripherie des Körpers oder vom Gehirne aus, die bei normalem Zustande des Nervensystems (Vasadilatatorenzentrums) 
weiter keine sichtbaren Folgen veranlassen, hier mehr minder schwere pathologische Veränderungen auf der Haut oder Schleimhaut auslösen. Daraus geht weiter hervor, daß wir den von den verschiedenen Autoren als Ursache für das Ulcus neuroticum (chron. Aphthen) angegebenen Momenten; traumatische Reize verschiedener Natur von der Schleimhaut des Mundes selbst, oder die bei den verschiedenen phisiologischen und pathologischen Zuständen des Genitalorganes (Menstruation, Gravidität, Laktation, ferner bei krankhaften Prozessen dieser und anderer Organe) von hier herrührenden, auf dem Wege des Zentralnervensystems auf obgenanntes Zentrum übergeleiteten Reizen, da 3 wir also diese Momente nicht als ursächliche, sondern bloß als auslösende Momente anzuerkennen vermögen, die bei Individuen, deren Vasadilatatorenzentrum durch irgendeine Ursache stärker labil ist, pathologische Zustände von der Art des Herpes oder einer Ulzeration auf der Schleimhaut auszulösen imstande sind; ob ein Herpes mucosae oder ein Ulcus mucosae entsteht, wird wohl hauptsächlich von der Intensität der Funktionsstörung des genannten Zentrums und andererseits von der Stärke des auslösenden peripheren oder zentralen Reizes abhängen.

Nach dieser Darstellung wäre also das Ulcus neuroticu m (ulcus aphthosum chronicum) aufzufassen als eine neurotische Schleimhautgangrän im Sinne der neurotischen Hautgangrän Kreibichs und wäre als eine Bereicherung der Zahl der von diesem Autor als auf angioneurotischer Grundlage beruhenden Affektionen (Herpes spl., Herpes zoster, Urtikaria, Prurigo etć.) anzusehen. 